\title{
凉山半细毛羊初生重性状分形特征分析
}

吴辉生, 吴登俊, 周明亮, 张翔宇

四川农业大学动物科技学院, 雅安 625014

摘要: 首次以非线性理论中的分形理论对凉山半细毛羊的初生重数据进行分析, 计算了 1996 2004 年间初生重 性状的信息维数、关联维数、无标度区及性状测度范围。结果表明: (1) 从 1996 2004 年凉山半细毛羊的初生 重性状的信息维数都集中在 0.66529 0.90675, 而且无标度区较大, 测度范围广。该结果说明, 每年羔羊初生重 信息维数都较大, 群体存在十分丰富的变异, 变异范围广, 具有十分巨大的育种潜力; (2) 相应的关联维数则集 中在 0.62438 0.86528 之间, 表明群体内个体遗传结构具有较强的相关性; (3) 这两个分形维数能够分别从两个 不同的角度揭示群体遗传结构的分形特征。

关键词：分形理论; 初生重; 凉山半细毛羊

\section{Analysis of the fractal properties of the birth weight of Liangshan semi-fine wool sheep}

\author{
WU Hui-Sheng, WU Deng-Jun, ZHOU Ming-Liang, ZHANG Xiang-Yu \\ College of Animal Science and Technology, Sichuan Agricultural University, Ya'an 625014, China
}

\begin{abstract}
Understanding of animal population genetic structure about quantitative traits is an important step in animal breeding. In the present study, the data of birth weight of the Liangshan semi-fine wool sheep was first studied by the fractal theory of nonlinear theory. The information dimension, correlation dimension, none-scale range, and the scale range were calculated. The results showed that (1) the information dimensions of the birth weight from 1996 to 2004 ranged from 0.66529 to 0.90675 . The none-scale range and the range of the variation were very large. This indicates that the population had large dimensions of the BW (birth weight), large variation, and great potential in breeding; (2) the correlation dimensions ranged from 0.62438 to 0.86528 , which indicates that the genetic structures of the population were highly correlated; (3) both of the two dimensions could reveal the fractal properties of the population genetic structure through two different aspects, and it was useful in studying the population genetic structure and animal breeding.
\end{abstract}

Keywords: fractal theory; birth weight; Liangshan semi-fine wool sheep

对于群体遗传结构和性状遗传变异行为的研究 是动物遗传育种研究中的重要组成部分, 是动物育 种的基础工作。通过对动物遗传结构和性状遗传变 异行为的研究, 可以分析种群的遗传变异程度, 评
估育种潜力, 从而为动物育种提供准确的指导。鉴 于动物的遗传结构与性状遗传变异行为, 在本质上 属于典型的非线性系统这一特点, 在其研究中运用 非线性理论成为了动物遗传育种研究中的一个重要

收稿日期: 2008-01-16; 修回日期: 2008-04-01

基金项目：四川省畜禽育种攻关项目(编号：01NG029-18)资助[Supported by Animal and Poultry Breeding Key Project in Sichuan Province of China (No. 01NG029-18)]

作者简介: 吴辉生(1983-), 男, 江西崇义人, 硕士, 专业方向 : 数量遗传与动物育种。E-mail: wuhs818@163.com

通讯作者：吴登俊(1956-)，男，四川名山人，教授，博士生导师，研究方向：动物分子遗传育种。Tel: 0835-2885848; E-mail: wdengjun@sicau.edu.cn 
方向。

世界的本质是非线性的，而分形是非线性的几 何表现。自 1975 年 Mandelbrot 第一次提出分形这 个概念以后, 在不同学科领域中分形被广泛地应用 起来, 1982 年, Mandelbrot 的“ The Fractal Geometry of nature" "[1]的出版, 标志着分形理论的初步形成, 逐渐成为了在自然科学和社会科学中处理复杂性和 非线性问题的强有力的理论工具。分形维数是分形 理论中最核心的概念与内容, 它是度量不规则物体 或分形体最主要的指标。分形维数不同, 物体内部 结构的复杂程度或它的动态演化过程就不同。它适 合于描述世界上内部结构的不同组成部分之间存在 相似性的不规则事物 ${ }^{[2]}$, 它为动物遗传结构与性状 变异的研究提供了一个新的途径。分形理论的创立 和发展为研究动物遗传的规律性建立了崭新的数学 语言和定量描述的方法, 同时为其提供了新思想和 新方法。

自然界中有很多物体在不同的观测角度都存在 一定的相关性, 如果它们的特性在较大的观测尺度 范围内具有相似性, 它们在这一尺度范围内就具有 自相似性并具有分形结构。自然界中大部分物体都 并非理想的分形体, 但在一定条件下却可以用分形 几何学的原理来描述它们。生物就是一种具有这种 特征的物质体。Havlin 等 ${ }^{[3]}$ 的研究揭示了在生物体 中广泛存在的分形特征, 而 Geoffrey 等 ${ }^{[4]}$ 以及 Williams ${ }^{[5]}$ 和 Weibel ${ }^{[6]}$ 的研究揭示并阐明了在生物中 存在的分形特征以及分形理论在生物学研究中的重 要性。分形维数是用来研究物种的空间分布的用力 工具 ${ }^{[7,8]}$, 在生态学研究中分形维数则已成为一种 常用的工具 ${ }^{[9,10]}$, 在群体遗传学的研究中分形理论 更是起了重要作用 ${ }^{[11,12]}$, 尤其是 Vlad ${ }^{[13]}$ 的研究更揭 示了在生物体遗传结构中分形规律的普遍性。这些 研究中分形理论的运用表明了在群体遗传结构与性 状遗传变异的研究中运用分形理论的可行性。虽然 在生物领域甚至群体遗传结构研究中分形理论运用 较广泛, 但以动物数量性状为对象运用分形理论进 行研究却尚未见报道。根据上述所述分形体的特征 判断, 将动物群体的数量性状整体当成一个分形体, 以分形维数对其进行研究, 考察其内部结构的复杂 程度或动态演化过程, 在理论上是可行的。本研究 首次运用非线性理论中的分形方法对凉山半细毛羊数 量性状的遗传变异特征进行分析, 以初生重(Birth weight, BW)性状为研究对象, 通过对其信息维数和
关联维数的计算, 分析该性状的遗传变异结构特征, 为其育种规划的制定及保种、选育和利用提供依据。 同时探讨了分形方法在动物数量性状的变异结构研 究中应用的可行性, 为在数量性状的研究中更广泛 地运用分形理论提供基础。

\section{1 材料和方法}

\section{1 材料来源}

本研究所用数据来源于凉山半细毛羊原种场。 共收集 1996 2004 年间出生的共 3336 只健康羔羊 的初生重记录,年度内饲养管理条件相同。

\section{2 数据分析}

\subsection{1初生重变异程度分析}

采用统计分析软件 SAS 的 GLM 程序对初生重 进行统计分析，并分析年度、性别、季节、单双羔 及胎次效应对初生重的影响。同时计算出各年份中 初生重的变异系数, 以各年份中的观测值最小值为 下限, 最大值为上限统计各年份的测度范围。

\subsection{2 初生重信息维数计算}

主要研究初生重数据的分形维数这一特征参数, 而在分形理论中对分形维数的计算方法根据不同的 需要, 有多种不同的计算公式, 而且对同一物体以 不同方式定义的分形维数各不相同。本研究主要采 用信息维数和关联维数进行分析, 根据文献 [14]所 述信息维数定义及原理, 计算过程如下:

首先将每年的初生重数据从小到大依次排列, 将其逐次从 2 等分划分到 20 等分, 得到一系列不同 的等分数据的点集分布。计算每一等分组中拥有的 个体数 $N i$, 若该年度总个体数为 $N$, 则该等分中的 概率为:

$$
P i=\frac{N i}{N}
$$

该等分中的信息量可计算为:

$$
I i=-P i \times \ln P i
$$

则划分为该等分时的总信息量为:

$$
I_{(\varepsilon)}=\sum I i
$$

则信息维数的计算公式为:

$$
D=-\lim _{\varepsilon \rightarrow 0} \frac{I_{(\varepsilon)}}{\ln \varepsilon}
$$

将每组等分中总信息量 $I(\varepsilon)$ 与之对应的等分距 离 $\varepsilon$ 在双对数坐标上进行直线拟合 (如果某一年度中 存在不同直线段区的则按不同区域的点集分布进行 
分段直线拟合), 目测判断散点图中呈明显直线区域 的点集分布, 该区域所得拟合直线斜率的绝对值即 为信息维数 $D$ 。

\subsection{3 初生重关联维数计算}

首先将群体内每一个体与所有其他个体间的欧 氏距离一一计算出来, 然后给定一个距离值, 查找 小于该距离的欧氏距离个数 $(\mathrm{Ni})$; 通过变换距离值, 可以得到一系列 $N i$, 因此小于某一距离值 $(\varepsilon)$ 的个 体所占比例为:

$$
C_{(\varepsilon)}=\frac{N i}{N}
$$

则依据关联维数的计算公式:

$$
D c=\lim _{\varepsilon \rightarrow 0} \frac{\lg C_{(\varepsilon)}}{\lg \varepsilon}
$$

将 $C_{(\varepsilon)}$ 与对应的距离值 $(\varepsilon)$ 在双对数坐标下进行 (分段) 直线拟合, 所得拟合直线的斜率的绝对值为 其关联维数。根据研究实际, 本研究确定距离值为 0 $\mathrm{kg}$ 到该年度最大距离值, 变换步长为 $0.05 \mathrm{~kg}$ 。

\section{3 统计分析软件}

应用统计分析软件 SAS(V9.0)及 R (R-2.6.1)语 言程序包对数据进行统计分析, 并进行分形维数的 计算。

\section{2 结果与分析}

\section{1 变异程度分析结果}

年度、性别、季节、单双羔及胎次对初生重性 状影响方差分析结果见表 1。结果显示, 除胎次对初 生重影响不显著 $(P>0.05)$ 外, 其余各因素对初生重 影响都极显著 $(P<0.01)$ 。初生重性状变异程度的分析 结果见表 2。从表 2 可看出初生重在不同的年份内 均存在一定的差异, 年度效应对初生重的影响极显 著 $(P<0.01)$ 。其中 2004 年的平均初生重最大, 达到 了 $3.8874 \pm 0.6493,2002$ 年的最小, 只有 $3.1699 \pm$ 0.4889 。计算的变异系数和测度范围可以作为群体 性状遗传变异程度的估计, 从表 2 可看出各年度间 变异系数存在一定的差异, 而这种差异可以认为是 由于群体变异丰富程度的不同造成的。其中变异系 数由大到小排列为 $1999>1996>1998>1997>2001>$ $2000>2004>2002>2003$ 。每一年度的测度范围都较 大, 变异范围都达到了 $2.2 \mathrm{~kg}$ 以上, 其中 1999 年的 变异范围最大, 从 $1.5 \mathrm{~kg}$ 到 $6.0 \mathrm{~kg}$, 总范围达到了 $4.5 \mathrm{~kg}$ 。
表 1 年度、季节、性别、单双羔及胎次对初生重影响的 显著性检验

Table 1 Significant difference test of the impact of years, season, sex, birth type, and parity on birth weight

\begin{tabular}{llc}
\hline \multicolumn{2}{c}{ 因素 } & $P$ 值检验 \\
\multicolumn{2}{c}{ Factor } & $P$ value \\
\hline 年度 & Year & $P<0.0001$ \\
季节 & Season & $P<0.0001$ \\
性别 & Sex & $P<0.01$ \\
单双羔 & Birth type & $P<0.0001$ \\
胎次 & Parity & NS \\
\hline
\end{tabular}

注：NS 表示不显著。

Note: NS denotes no significant difference.

表 2 羔羊初生重变异程度计算结果

Table 2 Variation of the birth weight of lambs

\begin{tabular}{cccc}
\hline $\begin{array}{c}\text { 年度 } \\
\text { Year }\end{array}$ & $\begin{array}{c}\text { 初生重 } \\
\text { Birth weight }\end{array}$ & $\begin{array}{c}\text { 变异系数 } \\
\text { C.V. }\end{array}$ & $\begin{array}{c}\text { 测度范围 } \\
\text { Scale range }\end{array}$ \\
\hline 1996 & $3.7343 \pm 0.8647^{\mathrm{Bb}}$ & 23.1562 & $1.80 \sim 5.80$ \\
1997 & $3.6278 \pm 0.8221^{\mathrm{Bb}}$ & 22.6613 & $1.20 \sim 5.40$ \\
1998 & $3.4435 \pm 0.7837^{\mathrm{C}}$ & 22.7591 & $1.50 \sim 6.00$ \\
1999 & $3.6076 \pm 0.9803^{\mathrm{Bb}}$ & 27.1730 & $1.40 \sim 6.50$ \\
2000 & $3.2093 \pm 0.6471^{\mathrm{Dd}}$ & 20.1649 & $1.20 \sim 5.10$ \\
2001 & $3.5532 \pm 0.7447^{\mathrm{BbC}}$ & 20.9594 & $1.40 \sim 5.00$ \\
2002 & $3.1699 \pm 0.4889^{\mathrm{Dd}}$ & 15.4228 & $1.80 \sim 5.10$ \\
2003 & $2.9601 \pm 0.3677^{\mathrm{E}}$ & 12.4215 & $2.00 \sim 4.20$ \\
2004 & $3.8874 \pm 0.6493^{\mathrm{A}}$ & 16.7025 & $2.50 \sim 6.50$ \\
\hline
\end{tabular}

注: 同一列中上标不同的小写字母表示差异达到显著水平 $(P<0.05)$, 上标不同的大写字母表示差异达到极显著水平 $(P<0.01)$ 。

Notes: Values followed by different letters within a row are significantly different at $P<0.05$ (the lowercase letters) or $P<0.01$ (the capital letters).

\section{2 凉山半细毛羊初生重性状的信息维数}

\section{2 .1 无标度区}

根据原始初生重数据经计算分析得出的 $I_{(\varepsilon)}-\ln (\varepsilon)$ 之间的关系如图 1 所示。图 1 显示，大部分年份 $\left(1996 、 1997 、 1998 、 1999 、 2003\right.$ 及 2004 年)的 $I_{(\varepsilon)}-\ln (\varepsilon)$ 之间在所有的观测尺度范围内都存在较好的线性规 律。但是 2000、2001 及 2002 年的线性规律在一定 的尺度上则会发生改变而成为另外一种线性规律, 而且不同年度拐点的位置也各不相同。这几年中从 拐点开始, 直线斜率趋于平缓, 绝对值有所下降。本 研究中确定以小于拐点的尺度范围作为无标度区, 以 该区域的直线斜率来估测该年度性状的信息维数。

\subsection{2 信息维数}

以拟合直线斜率的绝对值估测的各年度初生重 数据的信息维数结果如表 3 所示。从 1996 至 2004 年间的直线拟合相关系数绝对值大小均在 0.98 以上, 而且呈现极显著的线性相关。因而利用该方法求得 

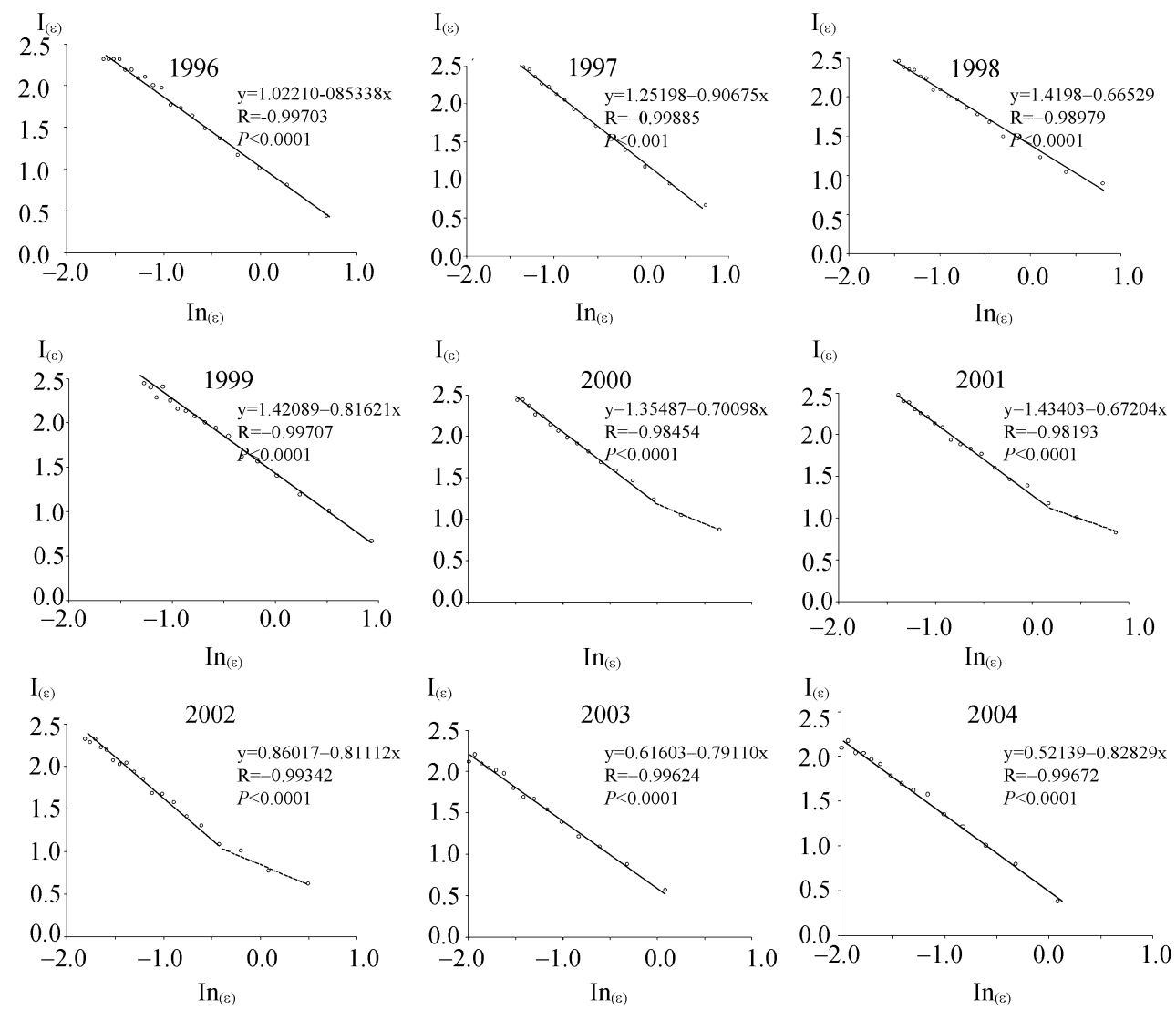

图 $11996 \sim 2004$ 年初生重 $I_{(\varepsilon)}-\ln _{(\varepsilon)}$ 分布函数图 实线所示为无标度区间, 直线斜率及相关系数为该区域点集计算结果。

Fig. $1 \quad I_{(\varepsilon)}-\ln _{(\varepsilon)}$ function of the birth weight from 1996 to 2004

Solid line shows the none-scale range. The slope and correlation dimension were calculated from this area.

的拟合直线斜率是真实可靠的。从结果可看出 1996 2004 年间凉山半细毛羊初生重这一数量性状, 群体结构信息维数在 0.66529 0.90675 之间, 变异显 著。最大的为 1997 年, $D$ 值达到了 0.90675 , 其次为 1996 年的 $0.85338, D$ 值最小的年份为 1998 年, 只有 0.66529 。每一年度的大小次序为 1997>1996>2004> $1999>2002>2003>2000>1998$ 。由图 1 及判断出的无 标度区中可看出每一年度中, 初生重 $D$ 值所适观测 尺度范围也较大。

\section{3 凉山半细毛羊初生重性状的关联维数}

\subsection{1无标度区}

由图 2 看出每一年度的 $\lg \varepsilon-\lg C_{(\varepsilon)}$ 关系都存在两个 不同线性规律的区域, 拐点的出现在本研究计算关联 维数过程中具有重要意义, 两个不同的尺度区域具有 不同的关联维数表明了在不同的尺度范围内个体存在 不同的关联程度, 因而本研究确定对每年度的两个不 同线性规律区域都进行关联维数的计算。
表 3 初生重性状信息维数计算结果

Table 3 The information dimension of the BW

\begin{tabular}{ccccc}
\hline $\begin{array}{c}\text { 年度 } \\
\text { Year }\end{array}$ & $\begin{array}{c}\text { 信息维数 } \\
\text { Information } \\
\text { dimension }\end{array}$ & $\begin{array}{c}\text { 相关系数 } \\
\text { Correlation } \\
\text { dimension }\end{array}$ & $\begin{array}{c}\text { 无标度区 } \\
\text { None-scale } \\
\text { range }\end{array}$ & $\begin{array}{c}\text { 显著水平 } \\
\text { Significant } \\
\text { level }(P)\end{array}$ \\
\hline 1996 & 0.85338 & -0.99703 & $0.20 \sim 2.00^{*}$ & $P<0.01$ \\
1997 & 0.90675 & -0.99885 & $0.21 \sim 2.10^{*}$ & $P<0.01$ \\
1998 & 0.66529 & -0.98979 & $0.23 \sim 2.25^{*}$ & $P<0.01$ \\
1999 & 0.81621 & -0.99707 & $0.26 \sim 2.55^{*}$ & $P<0.01$ \\
2000 & 0.70098 & -0.98454 & $0.20 \sim 1.00$ & $P<0.01$ \\
2001 & 0.67204 & -0.98193 & $0.24 \sim 2.40$ & $P<0.01$ \\
2002 & 0.81112 & -0.99342 & $0.17 \sim 0.66$ & $P<0.01$ \\
2003 & 0.79110 & -0.99624 & $0.11 \sim 1.10^{*}$ & $P<0.01$ \\
2004 & 0.82829 & -0.99672 & $0.11 \sim 1.10^{*}$ & $P<0.01$ \\
\hline
\end{tabular}

注: $*$ 表示该年度无标度区为整个测度尺度范围。

Note: * means that this none-scale range was distributed in the whole range of the scale size.

\subsection{2 关联维数}

通过拟合直线斜率的绝对值估测的各年度初生 重数据的关联维数结果如表 4 所示。其中每一年度 

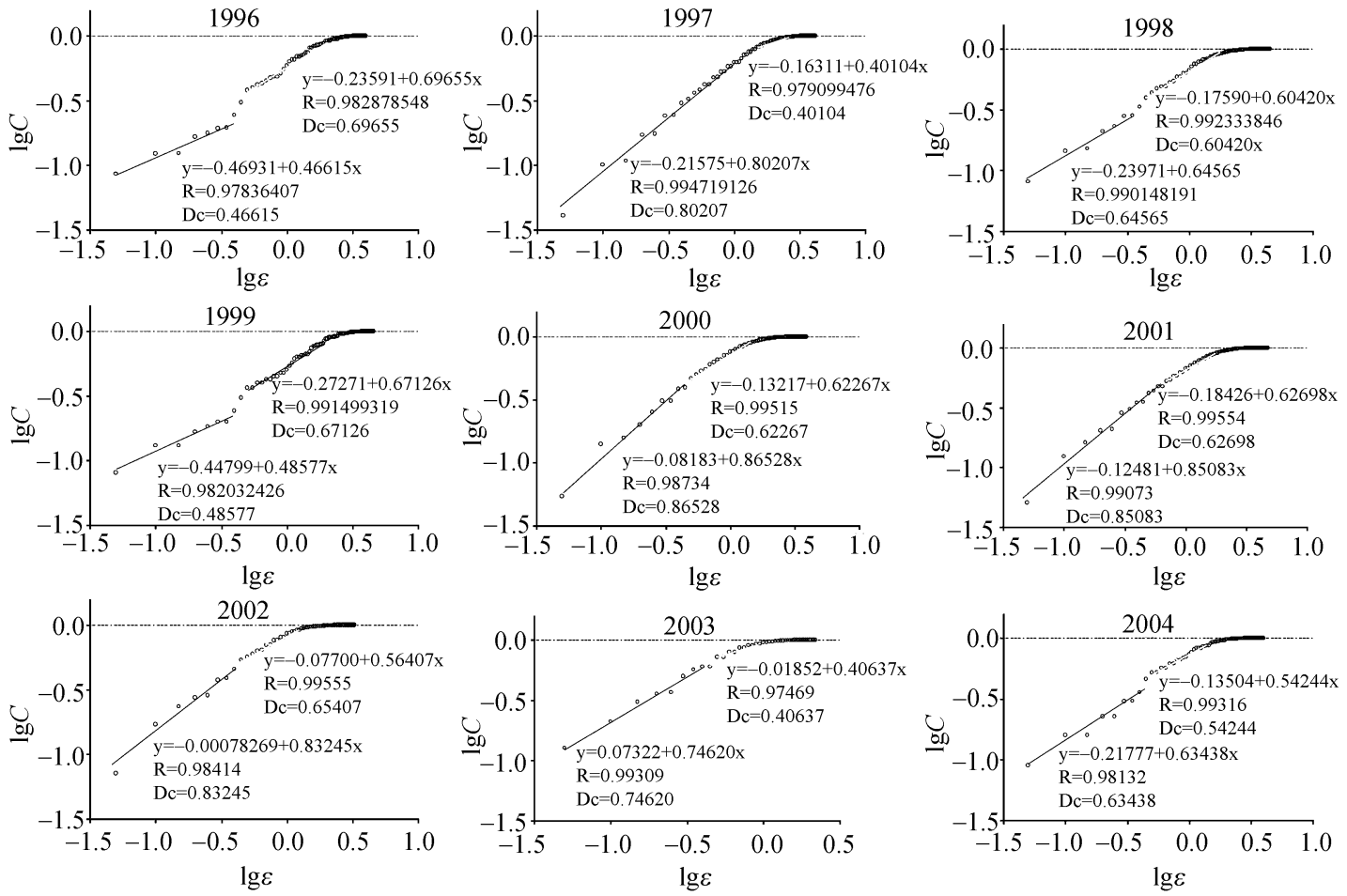

图 $21996 \sim 2004$ 年初生重 $\lg \varepsilon-\lg C_{(\varepsilon)}$ 分布函数图

Fig. $2 \lg \varepsilon-\lg C_{(\varepsilon)}$ function of the birth weight from 1996 to 2004

的初生重数据在两个不同的尺度范围内都存在两个 大小不同的关联维数, 并且直线拟合相关系数均在 0.97 以上, 而且呈现极显著的线性相关 $(P<0.01)$ 。因 而利用该方法拟合求得的两条直线斜率都是可靠 的。表 4 显示，除 1996 和 1999 年以外，其余年度的 初生重在较小尺度范围内的关联维数要大于在较大 尺度范围内的关联维数, 小尺度区域的关联维数揭 示了群体内个体相关的主要特征。大部分关联维数 都大于 0.5 , 这说明群体内个体间的遗传结构存在较 强的相关。

\section{3 讨 论}

\section{1 信息维数揭示的群体遗传结构的分形特征}

根据信息维数的定义, 计算信息维数过程中, 是通过信息量 I 来计算出每一尺度对其信息量的幂 律关系。信息 (又称负熵)是一个系统结构复杂性程 度的度量。系统结构的复杂性指的是系统组分分布 的非均匀性，即系统结构随测度而变化的程度 ${ }^{[15]}$ 。 一个系统结构信息量越少, 结构越简单, 反之信息 量越大, 其结构越复杂。因此从信息维数的大小可 以反应出随着观测尺度逐渐缩小, 性状信息量增加
程度的大小。而信息维数越大, 随着测度的缩小, 信息

表 4 初生重性状关联维数计算结果

Table 4 The correlation dimension of the birth weight

\begin{tabular}{cllcc}
\hline 年度 & $\begin{array}{c}\text { 关联维数 } \\
\text { Year } \\
\text { dimension }\end{array}$ & $\begin{array}{c}\text { 相关系数 } \\
\text { Correlation } \\
\text { dimension }\end{array}$ & $\begin{array}{c}\text { 显著水平 } \\
\text { Significant } \\
\text { level }\end{array}$ & $\begin{array}{c}\text { 尺度范围 } \\
\text { Scale range }\end{array}$ \\
\hline 1996 & 0.46615 & 0.97836 & $P<0.01$ & $0 \sim 0.35$ \\
& 0.69655 & 0.98288 & $P<0.01$ & $0.5 \sim 2.85$ \\
1997 & 0.80207 & 0.99472 & $P<0.01$ & $0 \sim 1.0$ \\
& 0.40104 & 0.97910 & $P<0.01$ & $1.45 \sim 2.80$ \\
1998 & 0.64565 & 0.99015 & $P<0.01$ & $0 \sim 0.35$ \\
& 0.60420 & 0.99233 & $P<0.01$ & $0.45 \sim 2.3$ \\
1999 & 0.48577 & 0.98203 & $P<0.01$ & $0 \sim 0.4$ \\
& 0.67126 & 0.99150 & $P<0.01$ & $0.5 \sim 2.95$ \\
2000 & 0.86528 & 0.98734 & $P<0.01$ & $0 \sim 0.45$ \\
& 0.62267 & 0.99515 & $P<0.01$ & $0.5 \sim 2.25$ \\
2001 & 0.85083 & 0.99073 & $P<0.01$ & $0 \sim 0.6$ \\
& 0.62698 & 0.99554 & $P<0.01$ & $0.7 \sim 2.3$ \\
2002 & 0.83245 & 0.98414 & $P<0.01$ & $0 \sim 0.4$ \\
& 0.65407 & 0.99555 & $P<0.01$ & $0.45 \sim 1.65$ \\
2003 & 0.74620 & 0.99309 & $P<0.01$ & $0 \sim 0.45$ \\
& 0.40637 & 0.97469 & $P<0.01$ & $0.5 \sim 1.35$ \\
2004 & 0.63438 & 0.98132 & $P<0.01$ & $0 \sim 0.4$ \\
& 0.54244 & 0.99316 & $P<0.01$ & $0.45 \sim 2.15$ \\
\hline & & & & \\
\hline
\end{tabular}


量增加得越快, 性状数值变化则越接近于连续分布。 当信息维数达到 1 时, 则系统结构复杂程度高, 该性 状随着测度的变化信息量变化大, 性状数值分布接 近于连续分布。因此在以信息维数来描述群体遗传 变异时, 信息维数越大, 则说明此群体在一定的测 度范围内, 当测度缩小到一定程度时, 性状数据点 集分布的变化越快, 此时, 该数量性状的变异潜力 较大, 在此变异群体中, 可供选择的变异个体较多。 而从生物统计学角度分析, 性状的变异系数和变异 范围可以在一定程度用来衡量该性状的变异程度。 因此将性状遗传变异的信息维数、变异系数及变异 范围结合起来分析, 可以对该性状的变异情况进行 准确的判断。

本研究中, 通过对 1996 2004 年 9 年间凉山半 细毛羊初生重这一数量性状进行信息维数的估测对 其分形特征进行研究, 结果表明, 此九年中所有年 份的羔羊初生重性状的信息维数都集中在 0.66529 0.90675之间, 都相对接近于 1, 这说明初 生重性状在测度发生变化时, 初生重数据的点集分 布的变化较快。当测度缩小时, 初生重性状的信息 量增加得快, 性状数据趋向于连续分布。而且每年 的无标度区间也较大。从表 1 可看出, 每年的变异 系数都达到了 0.98 以上, 而且以观测最大值、最小 值为上下限的测度范围也较大, 这表明群体每年的 羔羊初生重都具有十分丰富的变异存在, 变异范围 广, 从中可选择的差异明显的优良变异的机会大, 具有十分巨大的育种潜力。

表 3 的结果还表明年度间的信息维数存在一定 的波动, 这主要是因为年度效应的影响, 这与表 2 中各效应对初生重影响极显著的结果相一致。但是 信息维数的变化并不表现出明显的上升或下降的趋 势, 这主要是因为这几年的选育中都并未以初生重 作为主要的目标选择性状, 而年度间的差异主要是 由于年度效应中的气候等因素的影响形成的, 而且 每个年度中性别、季节、单双羔等各因素水平中数 据的不均衡性, 也可能对该年度的信息维数产生影 响, 从而使信息维数在年度间表现出差异, 但并不 表现出一定的规律性。

在分形理论中, 分形维数所适合的尺度范围被 称为无标度区, 无标度区是分形体表现自相似性的 尺度区间，在无标度区内，在不同的尺度上观察到 的分形体结构是相似的。在统计学意义上, 群体数 量性状的信息维数揭示出数量性状随着观测尺度的
变化其数据点集分布的变化规律。在拐点尺度前后, 整体信息量发生了较大变化, 表明该性状分布在不 同尺度上存在着显著差异。此时就需要判断哪一个 尺度区域的信息维数是合理的。因为信息维数反映 的是系统局域结构的非均匀性, 那么考察的尺度应 该是较小的, 否则就反映不出系统的微观结构特 征。因而本研究以小于拐点尺度的直线斜率来估测 该年度性状的信息维数是合理的。此无标度区度间 在本研究中的实际意义是在此区间内信息维数反映 出了该数量性状数据点集分布与观测尺度之间的幂 律关系。表 3 结果说明, 1996、1997、1998、1999、 2003 及 2004 年的无标度区为整个测度范围, 而 2000 年的为 $0.20 \sim 1.00,2001$ 年的为 $0.24 \sim 2.40,2002$ 年的为 $0.17 \sim 0.66$ 。这都说明该性状的无标度区间较 大, 在较大的观测尺度范围内, 数据点集分布与尺 度之间存在较好的幂律分布。而无标度区大, 这表 明初生重性状在很大的尺度范围内, 当尺度不断缩 小时, 总能得到新的分布特点。而在无标度区内, 该 性状的分布特点可由观测尺度按相应幂律关系加以 定量估测。但是对于没有检测出无标度区界限的数 据, 并不意味着它不存在标度界限。这只是说明该 群体所有个体数据点集分布未超越其无标度区范围, 如果考察尺度不断延伸, 则可逐渐检测到其无标度 区的边界 ${ }^{[12]}$ 。

\section{2 关联维数揭示的群体遗传结构的分形特征}

与信息维数不同, 关联维数从另一角度反映了 分形体的结构特征。相关函数 $C_{(\varepsilon)}$ 刻画了随着 $\varepsilon$ 的 增加, 点对数是如何增长的分布函数, 它在统计意 义上揭示出一个分形集合内部元素之间的关联程度, 表明了群体内部个体间的相关随尺度的变化程度。 计算群体内部的关联维数, 如果结果是在一定的尺 度域内只存在一个有意义的线性无标度区间，表明 种群内的所有个体数据的分布都遵从相同的规律, 这在统计意义上表明群体个体分布的均匀性。而本 研究的结果表明, 凉山半细毛羊初生重数据在计算 关联维数过程中, 会分别得到两个无标度区和两个 关联维数, 这说明群体内个体分布的非均匀性, 这 时两个关联维数都是有意义的, 此时大的关联维数 更能反映出群体内个体相关的主要特征, 可用它来 代表群体内部个体分布的关联维数。其中除 1996 年 和 2000 年外, 其余年度的关联维数都是在小尺度范 围内的值要大于大尺度范围的值。而这两年与其他 
年度不同的原因, 可能是因为本年度环境效应的作 用较大而引起的。关联维数越大, 表明群体内个体 的关联程度越高, 群体遗传结构的相关性越强。由 表 4 可看出, 各年度关联维数都集中在 $0.6 \sim 1.0$ 之间, 表明群体内个体的关联性较强, 生产性状分布均匀, 个体间的遗传结构存在较大的相关。

将各年度的关联维数和信息维数比较分析显示 (表 5), 1997 年的初生重数据分布聚集最强, 但平均 关联性稍弱, 分布不很均匀 (信息维数高, 关联维数 稍低), 即群体内存在着较丰富的遗传变异, 该数量 性状的变异潜力较大, 在此变异群体中, 可供选择 的变异个体最多, 但是群体内遗传结构相关性却较 弱。而 2000 年则是数据分布聚集较弱, 但是个体相 关性最强, 分布最均匀 (信息维数较低, 关联维数最 高), 即群体内遗传变异相对较少, 可供选择的变异 个体相对较少, 但是群体内遗传结构的相关性却最 强, 数据分布也最均匀。其它年份的两个分形维数 都各有差异, 分别揭示了在不同年度中个体分布的 聚集性、关联程度和分布的均匀性，这些都可作为 群体遗传结构分形特征的重要参数进行研究和分 析。可见, 这两个分形维数分别从不同的角度揭示 了群体的分形特征, 它们是相辅相承, 互相补充的。 这为了解群体的分形特征和遗传结构提供了丰富的 信息。对它们综合运用、从整体上解析它们的结构 组成将更有助于全面地了解群体的分形特征, 为群 体遗传结构的研究提供支持。

\section{表 51996 2004 年初生重性状的分形维数}

Table 5 The fractal dimension of the birth weight from 1996 to 2004

\begin{tabular}{ccc}
\hline $\begin{array}{c}\text { 年度 } \\
\text { Year }\end{array}$ & $\begin{array}{c}\text { 信息维数 } \\
\text { Information dimension }\end{array}$ & $\begin{array}{c}\text { 关联维数 } \\
\text { Correlation dimension }\end{array}$ \\
\hline 1996 & 0.85338 & 0.69655 \\
1997 & 0.90675 & 0.80207 \\
1998 & 0.66529 & 0.64565 \\
1999 & 0.81621 & 0.67126 \\
2000 & 0.70098 & 0.86528 \\
2001 & 0.67204 & 0.85083 \\
2002 & 0.81112 & 0.83245 \\
2003 & 0.79110 & 0.74620 \\
2004 & 0.82829 & 0.63438 \\
\hline
\end{tabular}

\section{参考文献(References):}

[1] Mandelbrot BB. The Fractal Geometry of Nature. New York: W H Freeman, 1982.

[2] Falconer KJ (ZENG Wen-Qu et al., translation). Fractal
Geometry: Mathematical Foundations and Applications. Shenyang: Northeast China University of Technology Press, 1991.

Falconer $\mathrm{KJ}$ (曾文曲等译). 分形理论—— 数学基础及 其应用. 沈阳: 东北工学院出版社, 1991.

[3] Havlin S, Buldyrev SV, Goldberger AL, Mantegna RN, Ossadnik SM, Peng CK, Simons M, Stanley HE. Fractals in biology and medicine. Chaos Solitons Fractals, 1995, 6(1): 171-201.[DOI]

[4] West GB, Brown JH, Enquist BJ. The fourth dimension of life: Fractal geometry and allometric scaling of organisms. Science, 1999, 284(5420): 1677-1679.[DOI]

[5] Williams N. Biology: fractal geometry gets the measure of life's scales. Science, 1997, 276(5309): 34-40.[DOI]

[6] Weibel ER. Fractal geometry: a design principle for living organisms. Am J Physiol Lung Cell Mol Physiol, 1991, 261(6): 361-369.

[7] Kunin WE. Extrapolating species abundance across spatial scales. Science, 1998, 281(5382): 1513-1515.[DOI]

[8] Cannas SA, Marco DE, Montemurro MA. Long range dispersal and spatial pattern formation in biological invasions. Math Biosci, 2006, 203(2): 155-170.[DOI]

[9] Halley JM, Hartley S, Kallimanis AS, Kunin WE, Lennon JJ, Sgardelis SP. Uses and abuses of fractal methodology in ecology. Ecol Lett, 2004, 7(3): 254-271.[DOI]

[10] MA Ke-Ming, ZU Yuan-Gang. Fractal properties of the spatial pattern of Larix gmelini population information dimension. Acta Ecol Sin, 2003, 20(2): 187-192.

马克明, 祖元刚. 兴安落叶松种群格局的分形特征-信 息维数. 生态学报, 2003, 20(2): 187-192.

[11] Paetkau D, Amstrup SC, Born EW, Calvert W, Derocher AE, Garner GW, Messier F, Stirling I, Taylor MK, Wiig O, Strobeck C. Genetic structure of the world's polar bear populations. Mol Ecol, 1999, 8(10): 1571-1584.[DOI]

[12] Wingen LU, Brown JKM, Shaw MW. The population genetic structure of clonal organisms generated by exponentially bounded and fat-tailed dispersal. Genetics, 2007, 177: 435-448.[DOI]

[13] Vlad MO, Morán F, Popa VT, Szedlacsek SE, Ross J. Functional, fractal nonlinear response with application to rate processes with memory, allometry, and population genetics. Proc Natl Acad Sci USA, 2007, 104(12): 4798-4803.[DOI]

[14] Dong Lian-Ke. Fractal Geometry and It's Applications. Shenyang: Liaoning Science and Technology Press, 1991, 25-30.

董连科. 分形理论及其应用. 沈阳: 辽宁科学技术出版 社. 1991, 25-30.

[15] ZU Yuan-Gang. Non-linear Ecological Modeling. Beijing: Science Press, 2004, 222-226.

祖元刚. 非线性生态模型. 北京: 科学出版社, 2004, 222-226. 